\title{
Fountain Codes and Linear Filtring to Mitigate Pilot Contamination Issue in Massive MiMo
}

\author{
Djedjiga Benzid $^{1} \&$ Miche Kadoch $^{1}$ \\ ${ }^{1}$ École de technologie supérieure, Montréal, Québec, Canada \\ Correspondence: Djedjiga Benzid, École de technologie supérieure, Montréal, Québec, Canada. E-mail: \\ djedjiga.benzid.1@ens.etsmtl.ca
}

Received: September 22, 2018 Accepted: October 8, 2018 Online Published: January 10, 2019

doi:10.5539/nct.v4n1p1 URL: https://doi.org/10.5539/nct.v4n1p1

\begin{abstract}
The fifth generation of cellular mobile $(5 \mathrm{G})$ is a future technology to meet growing capacity of users. For this prupose $5 \mathrm{G}$ will use advanced technologies. Very large multi- input multi- output or massive MiMo (m-MiMo) is considered as one of the promising technology. Nevertheless, the performance of m-MiMo is limited by pilot contamination issue. In fact, to mitigate pilot contamination issues in massive multi-input multi-output (m-MiMo), we proposed in previous work a new scheme where Raptor decoded symbols are used to estimate channel with Minimum Mean Square Error (MMSE) technique. The main benefit of this method is that the receiver does not need a transmitted pilot symbols to evaluate the channel, which allows saving power at transmission. The results showed that the MMSE scheme achieved the ideal case of the perfect channel. In this precedent paper, the MMSE detector and raptor code are used for their robustness among other schemes of linear detectors, and corrector codes, nevertheless, in case of m-MiMO, it was shown that all linear detectors work optimally. For this purpose, we include in this present article an additional linear filter to enhance the prior study, in which two supplementary detectors are considered, namely Zero Forcing (ZF) and Maximum Ratio Compression (MRC). The objective of this paper is to determine the ideal filtering technique and the robustness fountain code to address pilot contamination problem. In fact, the simulation results show that the ZF can attain the same ideal performance as the MMSE with raptor decoded symbols while MRC achieved lower performance compared to the other two shemes.
\end{abstract}

Keywords: 5G, massive MiMo, Raptor code, MMSE, ZF, LT code, LDPC code, Pilot contamination, CSI.

\section{Introduction}

To meet the users growing capacity of mobile cellular networks, a new generation of cellular mobile (5G) is planned for 2020, where advanced systems such m-MIMO are used to increase the performance and throughput of the wireless network. The massive MIMO is an enhancement of MIMO technology, involving hundreds of antennas. M-MIMO schemes are favourable for building the future digital society infrastructure; which will be categorized by their reliability, resilience and energy efficiency, making them able to connect human and objects users to the Internet and to other network infrastructure and clouds (Larsson, Edfors, Tufvesson, \& Marzetta, 2014). In fact, the advanced technology of MiMo can reduce interference and processing complexity of multi-user, and is desirable for eliminating thermal noise, fading and errors of channel estimation (Bogale \& Long Bao, 2014; Zirwas, Amin, \& Sternad, 2016). However, these advantages vanish against a major constraint affecting m-MiMo named pilot contamination(Marzetta, 2010); which is an interference resulted when processing the same pilot symbols in adjacent cells. Generally, to recover the received signals in multicell systems the knowledge of Channel State Information (CSI) is required at the base station (BS). Consequently, to evaluate the CSI, the BS receives an orthogonal pilot sequence from each user. However, in multicell networks, since the channel coherence interval is narrow, it is not possible to assign to a single-user an orthogonal pilots sequences, exclusively. Therefore, it required to reuse them from divers' cells, which generates interference between the received channel estimation in a specific cell and pilots transported by users of other cells.

This article is organised as follows: Sections 2 concerns a related works for previous research, sections 3 and 4 introduce the channel estimation schemes used in massive MiMo, and fountain codes respectively, section 5 describes the system model, in section 6 , we discuss our simulation results and finally, in section 7 , we give a conclusion of this paper. 


\section{Literature Review}

One way to fix pilot contamination problem is to avoid it by assuming that CSI is well-known at the receiver (Marzetta, 2010). However, in practice, it is impossible for the receivers to achieve a perfect CSI. Consequently, an estimation of channel is required to recover the received signal. Previous research work has proposed several solutions to diminish the effect of contaminated pilot symbols; one of the well-known propositions is named the Eigen Value Decomposition (SVD) method. However, it was shown that these channel estimation methods are greatly complex though using abundant antennas (Bogale \& Long Bao, 2014). To solve this problem, an iterative algorithm is proposed by the authors in the multiuser massive MiMo to enhance the pilot symbols and to evaluate the channel. Nevertheless, in this study, the authors suppose that the receiver has a perfect knowledge of CSI, which is difficult to obtain in practice. Another means to avoid the pilot interference issue is to estimate the channel by using the received data. The main advantage of this technique is that sender does not need to transmit the supplementary bits or pilot symbols in order to realize the analogous performance, thus the transmitter save the energy necessitated to transmit these pilots. The solution proposed by (Khoueiry \& Soleymani, 2014) is one of these estimator schemes; the main idea behind this proposition is to calculate the CSI when it is unavailable at the receiver. In this study the Raptor codes in slow Rayleigh fading channel is used to evaluate their scheme. Nevertheless, in this approach the authors neglect the Gaussian noise to estimate the CSI. In the same context, a novel system is proposed by the authors (Majumder \& Verma, 2013), where the raptor decoded information symbols are used to estimate CSI using Wiener filter estimator, however, this study is not dedicated for a huge quantity of antennas as in $\mathrm{m}$ - MiMo, in which detection schemes are different from Wiener filter. In order to fix the problem of symbols interference in m-MiMo, we proposed in previous work (Benzid \& Kadoch, 2018) a new scheme in which Raptor decoded symbols are used to estimate channel using (MMSE) as linear detectors in $\mathrm{m}$-MiMO where large number of antennas is used. Through this approach we proved that we can mitigate pilot contamination in m-MiMo using decoded symbols instead of the transmitted pilot symbols. Moreover, the linear detector MMSE is chosen for its robustness among other detectors. However, it's shown that in m-MiMo, where a large number of antennas are used, all linear detectors perform optimally. For this purpose, we include an alternative filtering scheme to the following study to complement the study of precedent paper, where an additional linear detection schemes namely, maximum ratio combining (MRC), zero-forcing receivers (ZF) are studied.

\section{Channel Estimation}

In wireless networks, to achieve transmission reliability, it is required to effectively recover symbols at the receiver. However, this latter suffers from interference. To reduce the impact of the inter symbols interference, the equalization techniques are used to remove the residues. One of the principal detection techniques used in practice is the maximum likelihood (ML) detector. This technique provides optimal performance to the receiver to detect all signals transmitted from the transmitter. However, it was shown that this technique has a complexity where it increases exponentially when the number of users increases. To reduce the decoding complexity of ML, the receiver can use a linear filtering on the received signal. Nevertheless, detection reliability of these schemes is inferior to those of ML detection. However, by using a large number of antennas, the linear detectors work optimally. Three schemes of linear detection are considered in this paper, the first one is the maximum ratio combining (MRC), while the second is zero-forcing receivers (ZF), and the third one is minimum mean-square error receivers (MMSE) as described below (Muaayed, 2017) (Ngo, 2015).

\subsection{Maximum-Ratio Combining (MRC)}

The main of MRC technique is to maximize the received signal-to-noise ratio (SNR) of each vector. The receiver multiplies the received stream with the conjugate transposes of the channel matrix $\mathrm{H}$, and then detects each vector separately which allows the MRC to have lower implementation complexity. However, beside this advantage the MRC performs poorly in scenarios where the interference is restricted.

\subsection{Zero-Forcing Receiver (ZF)}

The objective of zero-forcing (ZF) receivers is to avoid the impact of the contaminated pilots by using the pseudo-inverse of the channel gain matrix. Its advantage that its signal processing is simple. While the disadvantage of the ZF is that the pseudo-inverse can amplify the noise. If the interference between the symbols is important, the performance is deteriorating. Compared with the MRC, ZF has a higher complexity in terms of implementation because of the channel gain matrix computation.

\subsection{Minimum Mean Square Error (MMSE)}

To reduce symbol alteration, the MMSE scheme uses least squares technique between the evaluated random events and the intended events. Among other linear filters, the MMSE is considered the most efficient, because it 
maximizes the received SINR.

\section{Erasure and Rateless Codes}

In coding theory, an erasure code is a forward error correction (FEC) code which adds redundancy to the system to tolerate failures. This method does not require retransmission or feedback. The transmitter sends to the receiver data packets adding redundancy to the message; in this case the acknowledgement is not needed to confirm received packets. The receiver rejects any corrupted packet (Moreira \& Farrell, 2006). These codes are useful in the systems where retransmissions are costly or impossible. The rateless codes are characterized by non-fixed rate, the source generates a limitless number of encoded packets determined on the fly (MacKay, 2005). In fact, the transmitter floods the receiver with unlimited streams of coded bits, the receivers collect bits until they retrieve the content from the bits received, and then they send a STOP message to the transmitter.

\subsection{Low-Density Parity-Check (LDPC)}

Among the erasure codes, LDPC codes are the most important classes. Developed by Gallager in the early 1960s; based on iterative belief propagation (BP) algorithms decoding, which make them very powerful since they attain the Shannon limits in term of the decoding performance. LDPC codes perform better on additive white Gaussian noise (AWGN) channels and generally do not attain their optimum on non-Gaussian channels (Farrell \& Honary, 2005). The appearance of fountain codes makes it promising code for sparse graph code namely Luby Transform (LT), raptor. The most important applications of LDPC codes are the Internet or wireless networks (Farrell \& Honary, 2005).

\subsection{Fountain Code}

A fountain code also known as rateless-erasure codes, can be assimilated to water dropping from a fountain into a container, since the transmitter generates a continuous flow of transmitted data packets and the receiver collect data packet to recover the sent message.

\subsubsection{Luby Transformer (LT)}

Designed by Michael Luby in 1998, they are the first application of universal Fountain codes; its decoding is analogous to that used by the decoding LDPC.

\subsubsection{Raptor Codes}

Raptor codes are an enhancement of LT codes(Stockhammer, Shokrollahi, Watson, Luby, \& Gasiba, 2008), designed in the late 2000, and published in late 2001(Shokrollahi, 2006). In the Raptor codes, two concatenated coders are involved: a LT code, and an erasure coded, generally, the LDPC codes.

\section{System Model}

In this part of paper, the novel scheme is presented. Figure 1 illustrates the block diagram of the system. The scheme includes the transmitter which comprises a Raptor encoder and a BPSK modulator. The Raptor encoder is a succession of two encoders: an LDPC encoder and an LT encoder. The receiver includes the channel estimator followed by raptor decoder which containing an LT decoder and an LDPC decoder.

At the transmitter, the source generates a block message $s k \in$ (Jaber, Imran, Tafazolli, \& Tukmanov, 2016), $k=$ $\{0,1, \ldots . . \mathrm{K}-1\}$, sk is converted to the code word $b k \in$ (Amin, Zirwas, \& Haardt, 2015) of $k=0,1, \ldots \mathrm{L}-1$ bits. The BPSK is used modulate to generate the symbols vector $\quad x k \in\{-1,1\}, k=0,1 \ldots \ldots \mathrm{L}-1$. These symbols $x k$ are transferred over a slow flat fading channel. At the reception, the $y k$ signal is received at the linear filter node (channel estimator). The equation of received signal after estimation is given as follows:

$$
Y=H * X+N
$$




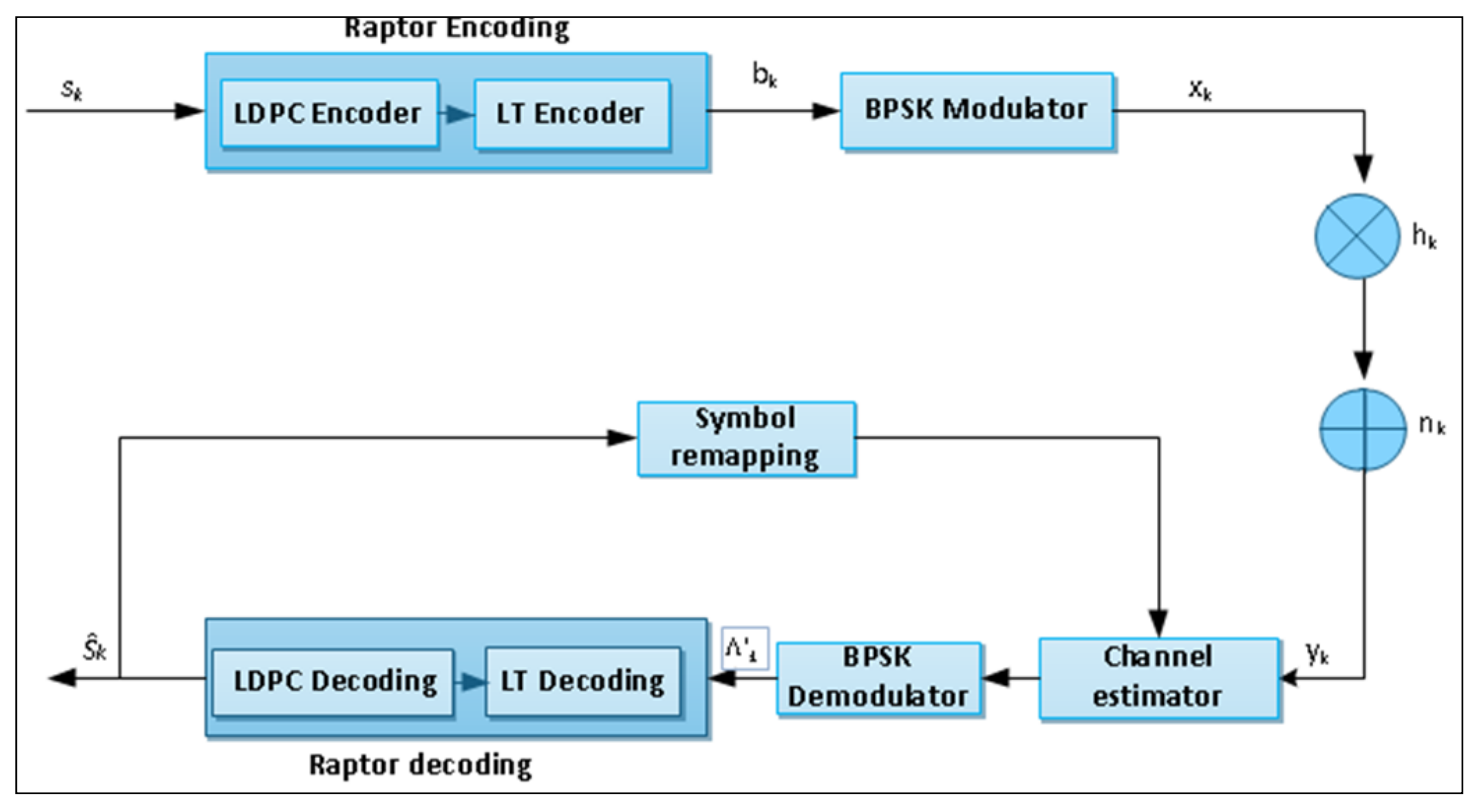

Figure 1. raptor diagram of estimate raptor decoded symbols with MMSE

$H$ is Channel matrix $\in \mathbb{C}^{N_{t} \times N_{r}}$, it defines the fading coefficients of the transmit antennas $\mathrm{Nt}$ and the receive antennas $\mathrm{Nr}$. The form of $\mathrm{H}$ is presented below:

$$
H=\left(\begin{array}{ccc}
h_{11} & \cdots & h_{1 N_{r}} \\
\vdots & \ddots & \vdots \\
h_{N_{t} 1} & \cdots & h_{N_{t} N_{r}}
\end{array}\right)
$$

It is considered that the coefficients of $\mathrm{H}$ are i.i.d, (independent and identically distributed) with zero mean and variance $\sigma_{h}^{2} . H$ is modelled as a Rayleigh random variable and its the probability density function (pdf) is specified by:

$$
p\left(h_{k}\right)=\frac{h_{k}}{\sigma_{h}^{2}} e^{\frac{h_{k}}{2 \sigma_{h}^{2}}}, h>0
$$

$\mathrm{Y}$ is received symbols vector, $\mathrm{X} \triangleq\left[\begin{array}{lll}x_{1} & \ldots & x_{k}\end{array}\right]^{T}$ is the vector of transmitted symbols and $N$ is the additive white Gaussian noise (AWGN) vector, with elements that are i.i.d, with 0 mean and variance $\sigma_{n}^{2}$.

\subsection{Channel Estimation}

In this section, we provide the most three estimate channel techniques using the decoded symbols.

\subsubsection{MMSE Channel Estimation}

Let $\hat{H}$ be an estimated random variable coefficient of $\mathrm{H}$, and $\mathrm{Y}$ be the observed random variable. $\mathrm{X}$ is transmitted pilot symbols. Assume that $R H Y$ is cross-covariance between $\mathrm{H}$ and $\mathrm{Y}$ and $R Y Y$ is covariance of $\mathrm{Y}$. Then, the estimated channel of $\mathrm{H}$ is given as follows:

$$
\begin{gathered}
\hat{H}=\left(R_{H Y} R_{Y Y}^{-1}\right) Y \\
R_{H Y}=E\left\{H Y^{T}\right\}=E\left\{H(H x+N)^{T}\right\}=E\left\{H H^{T} X^{T}\right\}+E\left\{\left(H N^{T}\right)\right\}
\end{gathered}
$$

$(\cdot)^{T}$ and $\mathrm{E}\{\cdot\}$ Denotes the matrix transpose and expected value respectively.

The term $E\left\{\left(H N^{T}\right)\right\}=0$ because $\mathrm{H}$ and $\mathrm{N}$ are i.i.d. Assume that $E\left\{H H^{T}\right\}=N_{t} \sigma_{h}^{2} I$ and, $E\left\{N N^{T}\right\}=N_{t} \sigma_{n}^{2} I$ 
$I$ is matrix identity, $\sigma_{n}$ is variance of noise signal and $\sigma_{h}$ is variance of the channel H. Since X does not change, we can state

$$
E\left\{H H^{T} X^{T}\right\}=X^{T} E\left\{H H^{T}\right\}=N_{t} \sigma_{h}^{2} X^{T}
$$

By Substituting (5) in (4) we have

$$
\begin{gathered}
R_{H Y}=N_{t} \sigma_{h}^{2} X^{T} \\
R_{Y Y}=E\left\{Y Y^{T}\right\}=X X^{T} E\left\{H H^{T}\right\}+E\left\{N N^{T}\right\}=X X^{T} N_{t} \sigma_{h}^{2} I+N_{t} \sigma^{2} I
\end{gathered}
$$

By Substituting (6) and (7) in (3) we have

$$
\hat{H}=\left(X X^{T}+\frac{\sigma_{n}^{2}}{\sigma_{h}^{2}} I\right)^{-1} X^{T} Y
$$

As discuss above, to resolve the issue of contaminated pilot symbols, raptor decoded information will be considered estimating CSI as an alternative to pilot symbols. Hence, pilot symbols $X$ are replaced by $\hat{\mathrm{S}}$ in Equation (8) of MMSE estimation, consequently the estimated channel $\hat{H}$ is expressed as shown below in Equation (9):

$$
\hat{H}=\left(\hat{S} \hat{S}^{T}+\frac{\sigma_{n}^{2}}{\sigma_{h}^{2}} I\right)^{-1} \hat{S}^{T} Y
$$

\subsubsection{ZF Channel Estimation}

The goal behind using Zero Forcing is to minimize the error vector. The error vector is the norm of $Y-H X$.

$\mathrm{Y}$ is the measurement or observed random variable. $\mathrm{X}$ is transmitted pilot symbols and $\mathrm{H}$ are the unknown random variable coefficients vector. Let $\hat{H}$ be estimated random variable coefficients of $\mathrm{H}$, and $\mathrm{F}$ be error vector, $\mathrm{F}$ is expressed as follows:

$$
F=\|Y-H X\|^{2}=(\mathrm{Y}-\mathrm{HX})^{\mathrm{T}}(\mathrm{Y}-\mathrm{HX})
$$

To minimize the error $\mathrm{F}$ we use a vector differentiation $\frac{d F}{d H}$ and we set the derivative equals to 0 so that Equation (9) becomes as follows:

$$
\frac{d F}{d H}=0 \Rightarrow-2 X^{T} Y+X^{T} H X=0
$$

By resolving (10), the estimate of $\mathrm{H}$ for $\mathrm{ZF}$ scheme is given as

$$
\hat{H}=X^{T} Y\left(X^{T} X\right)
$$

As done in subsection 5.1.1, since the information decoded symbols $\hat{S}$ are used as a substitute to pilot symbols X the Equation (12) becomes as follows:

$$
\hat{H}=\left(\hat{S} \hat{S}^{T}\right)^{-1} \hat{S}^{T} Y
$$

We can note that in high SNR when the $\sigma_{\mathrm{n}}$ tends 0 , equation (9) of the MMSE channel estimate becomes similar to of the ZF channel estimate Equation (13).

\subsubsection{MRC Channel Estimation}

The filtering in MRC indicated by multiplying the received signal by the conjugate transpose of the channel estimate. In our case we use MMSE channel estimate, Hence From (1), (9), we have

$$
\tilde{Y}=\hat{H}^{T} * Y
$$


Let $\hat{h}_{j}^{T}$ be the $\mathrm{j}$ th element of $\hat{H}, \hat{H}$ is MMSE channel estimate calculated in (9), so $\hat{h}_{j}$ is expressed as:

$$
\hat{h}_{j}^{T}=\left(\left(\hat{S}_{j} \hat{S}_{j}^{T}+\frac{\sigma_{n}^{2}}{\sigma_{h}^{2}} I\right)^{-1} \hat{S}_{j} Y_{j}\right)^{T}
$$

By substituting (14) in (13) we get:

$$
\tilde{Y}_{j+1}=\hat{h}_{j}^{T} Y_{j+1}=\left(\left(\hat{S}_{j} \hat{S}_{j}^{T}+\frac{\sigma_{n}^{2}}{\sigma_{h}^{2}} I\right)^{-1} \hat{S}_{j} Y_{j}\right)^{T} Y_{j+1}
$$

\subsection{Decoding}

After the channel estimation, the soft decoding process is achieved using the belief propagation (BP). The messages are passed between the $o$ variable nodes and the check nodes $i$. The likelihood ratios (LLR) of channels for each coded bit are stated as follows:

$$
Z_{0}=\ln \left(\frac{P\left(\hat{s}_{k}=1 \mid y_{k}, h_{k}\right)}{P\left(\hat{s}_{k}=-1 \mid y_{k}, h_{k}\right)}\right)
$$

By employing the independence property between $\hat{\mathrm{S}}_{\mathrm{k}}$ and $\hat{h}_{k}$, and using the Bayes rule the equation (17) becomes as follows:

$$
Z_{0}=\ln \left(\frac{P\left(y_{k} \mid \mathrm{h}_{k}, \hat{s}_{k}=1\right)}{P\left(y_{k} \mid \mathrm{h}_{k}, \hat{s}_{k}=-1\right)}\right)+\ln \left(\frac{P\left(\hat{s}_{k}=1\right)}{P\left(\hat{s}_{k}=-1\right)}\right)
$$

With equal probability for the input $S$, the term on the right side of the Equation (17) is equal to zero. In the output of the matched filter, $\mathrm{y}_{\mathrm{k}}$, the probability is given as follows:

$$
P\left(y_{k} \mid \mathrm{h}_{k}, \hat{s}_{k}= \pm 1\right)=\frac{1}{\sigma_{n} \sqrt{2 \pi}} e^{\frac{\left(y_{k} \pm h_{k}\right)^{2}}{2 \sigma_{n}^{2}}}
$$

By Substituting (19) in (18), we have

$$
Z_{0}=\frac{2 \hat{h}_{k}}{\sigma_{n}^{2 ?}} y_{k}
$$

In the first iteration of decoding, the estimated channel can be calculated, so $Z_{0}$ expressed as $Z_{0}=\frac{2}{\sigma_{n}^{2 ?}} y_{k}$

\subsubsection{Raptor Decoding}

Figure 2 shows the decoding graph of raptor code. At the iteration 0 of the BP decoding algorithm, if $o$ and $i$ are neighbours, the received channel LLR from the output node $o$ to the input node $i$ is expressed as follows:

$$
m_{o, i}^{(0)}=Z_{0}
$$

for the following iterations, $l=1, \cdots, N^{\text {itr }}$, the LLR updating process of LT decoding is completed as follows (Etesami \& Shokrollahi, 2006)

$$
m_{i o}^{(l)}=\sum_{o^{\prime} \neq o} m_{o^{\prime} i}^{l-1}
$$




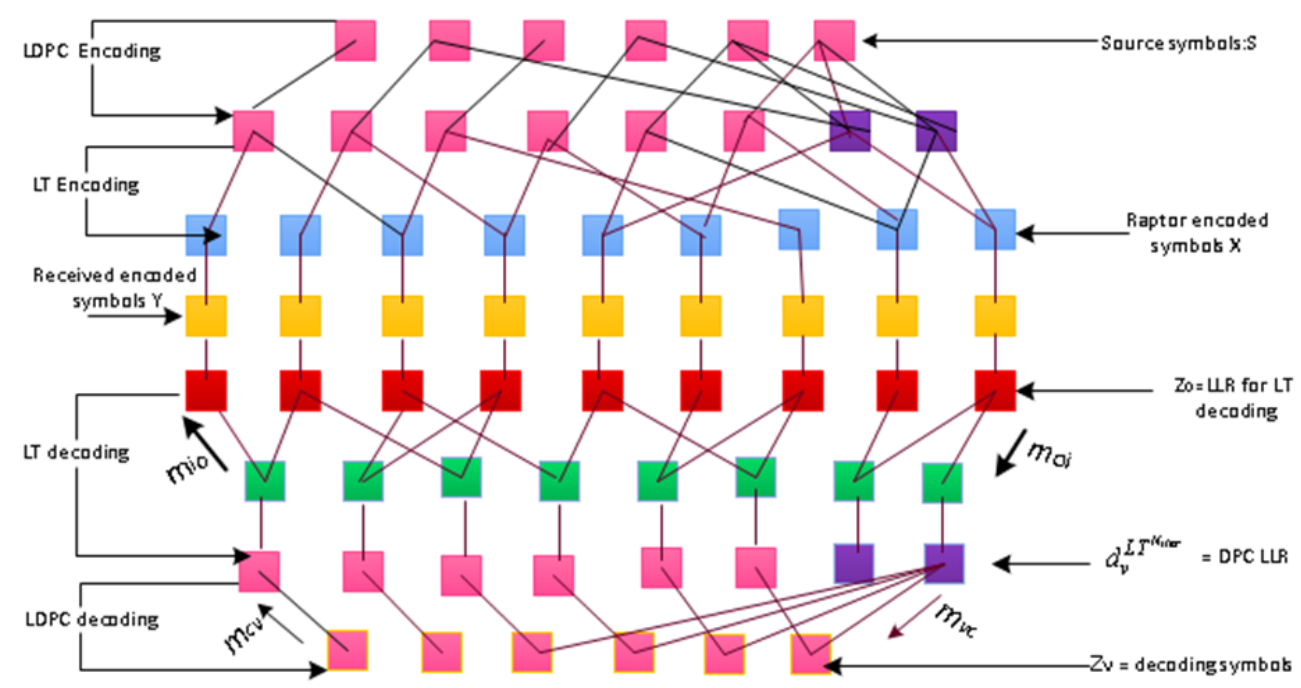

Figure 2. the decoding graph of Raptor code

$$
\tanh \left(\frac{m_{o, i}^{(l)}}{2}\right)=\tanh \left(\frac{z_{0}}{2}\right) \prod_{i^{\prime} \neq i} \tanh \left(\frac{m_{i^{\prime}, o}^{(l)}}{2}\right) \quad o=1, \ldots, \mathrm{L}
$$

$m_{o, i}^{(l)}$ and $m_{i o}^{(l)}$ are the messages at iteration $l$, spent from the output node $o$ to the input node $i$ and from the input node $i$ to the output node $o$, respectively. ${ }^{z_{0}}$ is the LLR corresponding to output symbol $o$ calculated in (17) and received from the channel. After processing the decoder for $l$ iterations, the LLR of each input node $i$ is given bellow:

$$
d_{i}^{L T^{l}}=\sum_{o \in P(i)} m_{o i}^{l}
$$

At iteration $N^{i t r}$, the LLR of the input nodes is calculated, as:

$$
d_{i}^{L T^{N_{\text {iter }}}}=\sum_{o \in P(i)} m_{o i}^{l}
$$

Where, ${ }^{P(i)}$, is the sum of overall output bits $o$ adjacent to $i$. Those LLR, named the output LLR, are the LT decoding LLR, considered as a priori LLR used as an input of the LDPC-decoding.

At iteration 0 of the algorithm, the messages sent by each variable node to its adjacent check nodes is the LLR from the LT decoding. The procedures of the LLR update for decoding LDPC are given by:

$$
\begin{gathered}
m_{v, c}^{(0)}=d_{v}^{L T^{N^{i t e r}}} \text { if o and i are neighbours } \\
\tanh \left(\frac{m_{c, v}^{(l)}}{2}\right)=\prod_{\substack{v=1 \\
v^{\prime} \neq v}}^{n} \tanh \left(\frac{m_{v^{\prime}, c}^{(l-1)}}{2}\right) \\
m_{v c}^{l}=m_{v, c}^{(0)}+\sum_{c^{\prime} \neq c} m_{c^{\prime}, v}^{l-1}
\end{gathered}
$$

$m_{v c}^{l}$ and $m_{c, v}^{l}$ are the messages of the LDPC decoder, they are transferred from the variable nodes $v$ to the check nodes $c$ and from the check nodes $c$ to the variables nodes $v$, respectively. At the iteration $l$, at the LDPC decoder, 
we get:

$$
Z_{v}=\sum m_{c, v}^{l}
$$

For each decoded bit c, $v$, hard decision is made as follows:

$$
\hat{S}=\left\{\begin{array}{l}
0 \text { if } Z_{v} \geq 0 \\
1 \text { if } Z_{v}<0
\end{array}\right\}
$$

After the hard decision, the estimate channel $\hat{H}$ is recalculated using (9).

\subsubsection{LT Decoding}

The belief propagation (BP) algorithm is used to achieve the soft decoding process. LT decoding is consisting of LDPC decoding. At the iteration 0 of BP decoding algorithm, if $o$ and $i$ are neighbours, then the received channel LLR from the output node $o$ to the input node $i$ is expressed as follows:

$$
\begin{gathered}
m_{o, i}^{(0)}=Z_{0} \\
\tanh \left(\frac{m_{o, i}^{(l)}}{2}\right)=\tanh \left(\frac{z_{0}}{2}\right) \prod_{i^{\prime} \neq i} \tanh \left(\frac{m_{i^{\prime}, o}^{(l-1)}}{2}\right)
\end{gathered}
$$

Variable nodes updating at $1=1, \cdots, N^{i t r}$ is given below:

$$
m_{i o}^{(l+1)}=\sum_{o^{\prime} \neq o} m_{o^{\prime} i}^{l}
$$

At $N^{\text {itr }}$ iteration, the LLR of each variable node is computed as:

$$
m_{i o}^{N_{i t e r}}=\sum_{o^{\prime} \neq 0} m_{o^{\prime} i}^{l}
$$

For each decoded bit $o, i$, the hard decision is made as follows:

$$
\hat{S}=\left\{\begin{array}{lll}
0, & \text { if } & m_{i o}^{N_{\text {iter }}} \leq 0 \\
1, & \text { if } & m_{\text {io }}^{N_{\text {iter }}} \geq 0
\end{array}\right\}
$$

\subsubsection{LDPC Decoding}

At the iteration 0 of BP decoding algorithm, if o and i are neighbours, the received channel LLR from the output node $o$ to the input node $i$ is expressed as follows:

$$
\begin{gathered}
m_{o, i}^{(0)}=Z_{0} \\
\tanh \left(\frac{m_{o, i}^{(l)}}{2}\right)=\tanh \left(\frac{m_{i^{\prime}, o}^{(l)}}{2}\right)
\end{gathered}
$$

At $l$ iteration, $l=1, \cdots N^{\text {it }}$, to update variable nodes, the message from LDPC variable nodes to check nodes is calculated as:

$$
m_{i o}^{(l+1)}=Z_{0}+\sum_{o^{\prime} \neq o} m_{o^{\prime} i}^{l}
$$

At $N^{\text {itr }}$ iteration, a posteriori log-likelihood ratio is done, the decision information of each variable node is computed as given below:

$$
m_{i o}^{N^{i t r}}=Z_{0}+\sum_{o^{\prime} \neq o} m_{o^{\prime} i}^{l}
$$

For each decoded bit $o, i$, hard decision is made as follows: 


$$
\hat{S}=\left\{\begin{array}{lll}
0, & \text { if } & m_{i o}^{N^{i t r}} \leq 0 \\
1, & \text { if } & m_{i o}^{N^{i t r}} \geq 0
\end{array}\right\}
$$

\section{Simulation and Results}

In this part of the paper, numerical results of the proposed scheme are presented. The system is evaluated with a massive MIMO involving 16 antennas at receiver and transmitter. A rate at 0.98 of raptor code is considered. A LT code and LDPC code degree distribution used in (Majumder \& Verma, 2013) is studied in this article. Achieved results are compared to the other approaches found in the literature and to regular estimator systems. In this paper we use the term of regular estimator systems to indicate that the detectors do not use decoded information to estimate channel.

\subsection{Proposed Channel Estimation Using MMSE and Raptor Code}

Three scenarios are studied in this subsection, firstly the perfect CSI, secondly our proposed scheme and thirdly no SCI available at the receiver. Raptor decoded symbols are used to estimate MMSE channel scheme, the length $N$ of code word considered is 800000 bits and message length $K$ is 7840 bits code. As revealed by the graphs of the Figure 3. The new system (showed in blue color) achieves the ideal case when state of the channel is available at the receiver (Perfect CSI graph) with a Bit Error Ratio (BER) equal to ${ }^{10^{-6}}$. Related to case when the CSI is unknown at the receiver (graph presented in magenta color), our scheme performs

better since we acquire lower rates of SNR thresholds and BER. By comparing our results to that found in (Khoueiry \& Soleymani, 2014), we realize that the threshold value is similar to the one obtained in (Khoueiry \& Soleymani, 2014), however, with a proposed scheme we achieve a smaller BER than (Khoueiry \& Soleymani, 2014).

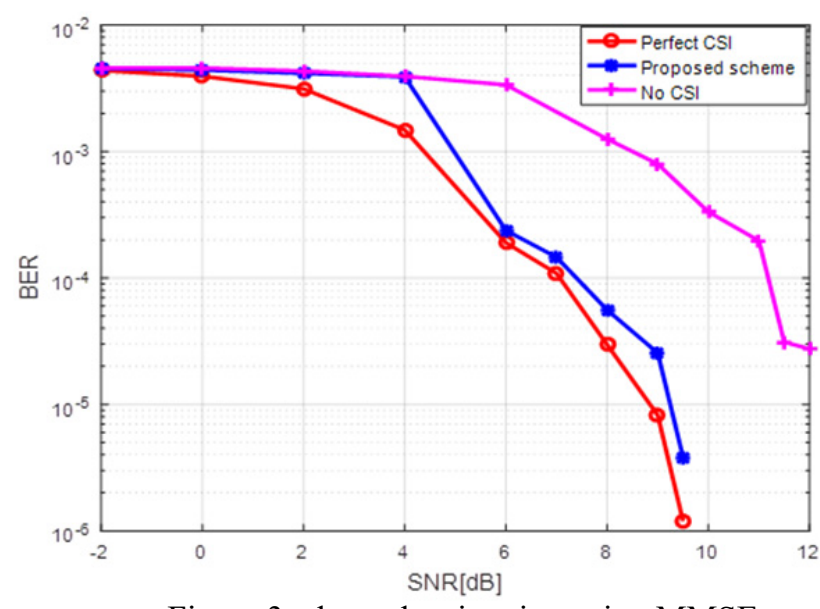

Figure 3. channel estimation using MMSE with Raptor decoded symbols with

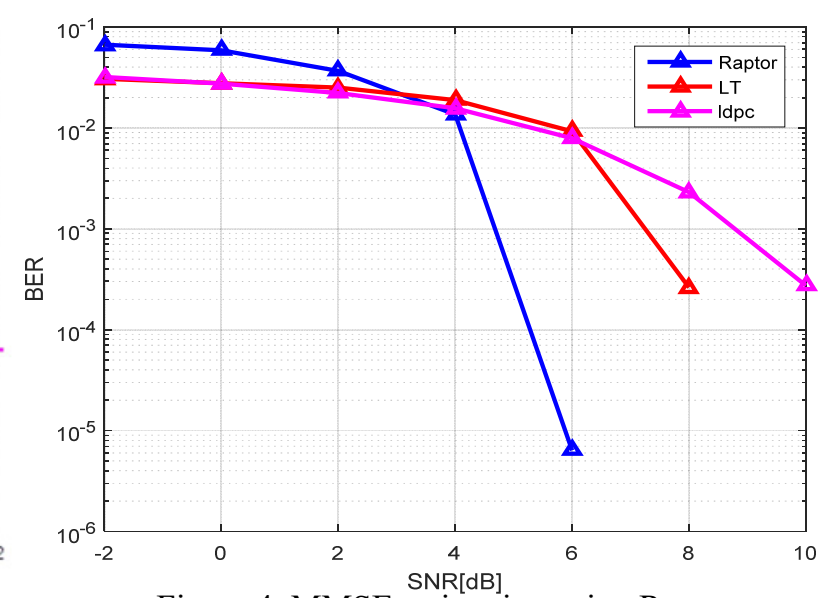

Figure 4. MMSE estimation using Raptor

\subsection{Proposed Channel Estimation Approach Using MMSE, LT and LDPC Codes}

In this subsection, our proposed scheme is simulated with the LT and the LDPC decoded symbols using MMSE detector. The simulation uses the length $K$ of code word of 15860, the length $N$ of the message is 160000 bits, and the number of the transmitted and received antennas are same as in section 6.1. The graphs of Figure 4 show that raptor code performs better than the LT and the LDPC using MMSE. The raptor code performs about $2 \mathrm{~dB}$ from the LT and $4 \mathrm{~dB}$ from LDPC. The LDPC codes achieve worst values of BER, because these latter are designed to be used on additive white Gaussian noise (AWGN) channels and do not perform well on non-Gaussian channels(Farrell \& Honary, 2005). Raptor codes perform better than LT because their structure and characteristics of rateless codes permit them to have a great capability of correcting errors.

\subsection{Proposed Channel estimation Approach Using Raptor Code, MMSE, ZF and MRC}

Further to the study done with MMSE filtering scheme, we consider in this subsection two additional filtering system, ZF and MRC, which are simulated using raptor decoded symbols. In this simulation we estimate a message with length $K$ equal to 16000 bits, the length $N$ of code word is 160000 bits, the number of the transmitted 
and received antennas is same as 6.2. As it is shown in Figure 5, the raptor code does not perform the same BER and the same SNR threshold as in section 6.1. This is due to that scheme in this subsection (6.3) is simulated with a decoding number iteration and a length code lower than those used in section 6.1. The graphs show that the system with raptor decoded symbols using both ZF MMSE have the same performance, which perform better than that using MRC technique. We obtain the same performance for ZF and MMSE because the number of antennas is high which allow ZF to get high SNR, as it explained in section 5.1.1, at high SNR, MMSE and ZF perform similar coefficients of channel estimate, hence they achieve the same performance. Due to these similarities between ZF and MMSE, in terms of performance, for the following simulation we focus our study only on ZF and MMSE.

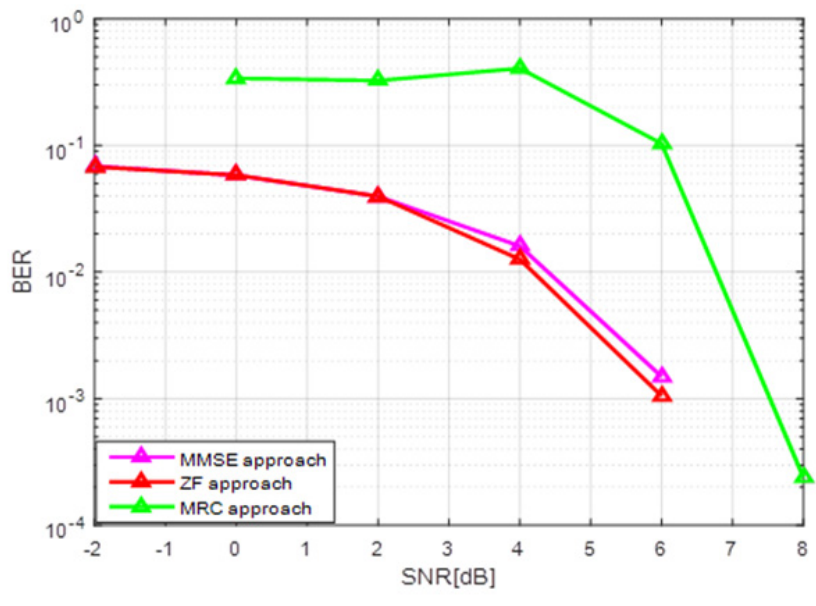

Figure 5. MMSE, ZF and MRC estimator using Raptor decoded symbols

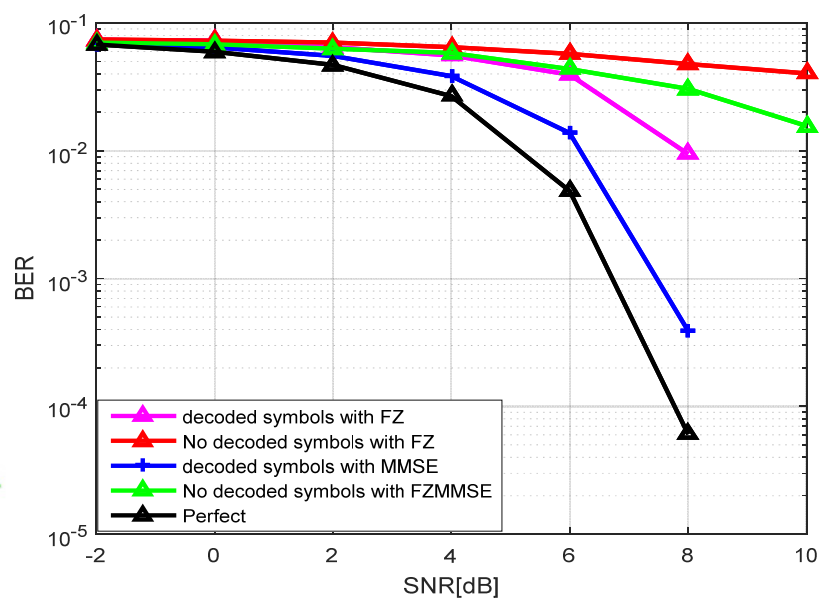

Figure 6. Channel estimation using MMSE with Raptor decoded symbols with

\subsection{Study of the Channel Estimation With The Regular Detector Systems: MMSE and ZF}

In this subsection we consider the case when the channel is estimed with received symbols using regular MMSE and ZF filtres. Which means that Raptor code will be used to recover the information but will not be used to estimate the channel. We name this scenario estimation channel with no decoded information. The result will be compared to those obtained in our proposed scheme, when decoded symbols are used get CSI.

In this simulation we estimate a message with length $K$ equal to 16000 bits, the length $N$ of code word is 160000 bits, the number of the transmitted and received antennas is same as those found in the section 6.2. The graphs of Figure 6. show that ZF and MMSE reach the same value of SNR threshold at $8 \mathrm{~dB}$ using decoded symbols to estimate the channel. However, by using the no decoded symbols their performance is deterioring, since they perform lower SNR threshold. This result is coherent, since the decoded information are recovered with less errors, and by using them to estimate the channel, their reliability add to the detectors schemes the robustness.

\subsection{Study of the Channel Estimation Using LT Code and Regular Estimator: MMSE and ZF}

In this sub-section, the scenario of LT decoded information over AWGN channel is simulated and compared to the LT over fading channel using decoded informations and no decoded information. The channel is estimated with MMSE and ZF techniques. In this simulation we estimate a message with length $K$ equal to 16000 bits, the length $N$ of code word is 160000 bits, the number of the transmitted and received antennas is same as 6.2.

As we can see in Figure 7, LT over AWGN channel (represented by blue color) and LT over fading channel estimated with MMSE decoded symbols, perform the same value of BER and SNR threshold, However, the LT code over fading channel with no decoded information, estimated with MMSE and ZF, perform less than that found with LT decoded information, since it achieves about $2 \mathrm{~dB}$ from the value of the SNR threshold using no decoded information. This result is coherent since decoded information is containg less erros than no decoded symbols. We can notice that by using LT code, the MMSE and ZF do not achieve the same performance as when they are used in Raptor codes. This is because the Raptor code has a great ability to correct errors, by using it to estimate the channel it enhances the performance of ZF which should work optimally when the number of antennas is great.

\subsection{Study of the Channel Estimation Using LDPCcode and a Regular Estimator: MMSE and ZF}

This section, the scenario of LDPC decoded information over AWGN channel is simulated and compared to the 
LDPC over fading channel with decoded informations and no decoded information using MMSE and ZF estimation. As it is shown at Figure 8, LDPC over AWGN channel (represented by green color) performs better than LDPC over fading channel. As discuss in above sections, the LDPC perform well in (AWGN) channels beause they are designed to be used on additive white Gaussian noise and do not perform well on non-Gaussian channels (Farrell \& Honary, 2005). We constate by using both MMSE and ZF, the LDPC over fading channel with decoded information performs better than that LDPC no decoded information. Since the decoded information are corrected, they became reliable, which gives them more robustess.

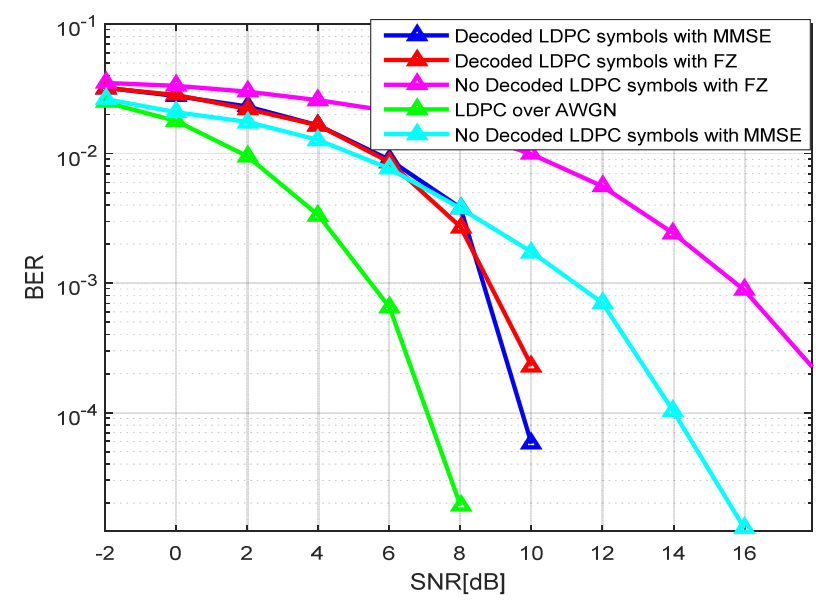

Figure 7. LT over AWGN and fading channel with decoded information and no decoded information using MMSE and $\mathrm{ZF}$

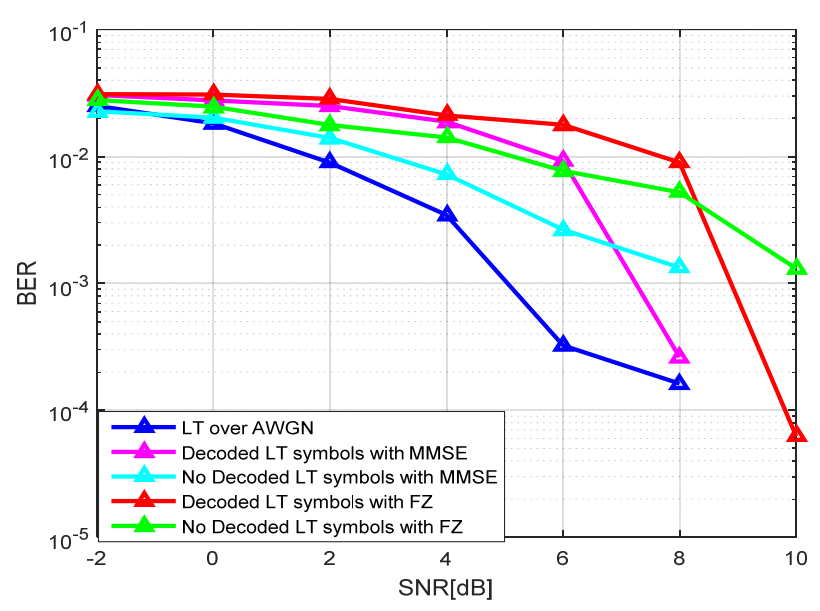

Figure 8. LDPC over AWGN and fading channel with decoded information and no decoded information using MMSE and ZF

\section{Conclusion}

In this article a new scheme to resolve a pilot contamination problem in m-Mimo is presented. The main idea behind this approach is to use symbols after decoding as an alternative to the transmitted pilot symbols to estimate channel. In previous work we studied MMSE channel estimation with raptor decoded symbols to evaluate the CSI in m-MiMO. To enhance our research, we extend our work to include other linear detector to estimate channel and other corrector codes to obtain symbols decoded. The LT and LDPC are included to this study to obtain correct information as well as ZF and MRC to evaluate the channel. Our approach is evaluated by simulating several scenarios. Numerical results show that, by using a large number of antennas, MMSE and ZF achieve the similar performance. Through this study, we show that the raptor decoded symbols are the most performant against LDPC and LT schemes for estimating the channel. Hence, by proving that using raptor decoded symbol with linear filtering can prevent from pilot to be contamined in m-MiMo. The main advantage of the proposed approach that it saves more energy since the symbols pilot are not required at receiver which decreases the power consumption at the transmit antennas. Another benefit for new system is that it is robust and reliable as it achieves smaller BER 
compared to other methods of channel estimation. Finally by using corrector code to estimate channel we avoid the network overhead because the corrector code adds the redundancy at the sent packet, consequently retransmission or feedback are not required.

\section{References}

Amin, M. B., Zirwas, W., \& Haardt, M. (2015). Advanced channel prediction concepts for $5 G$ radio systems. Paper presented at the 2015 International Symposium on Wireless Communication Systems (ISWCS). https://doi.org/10.1109/ISWCS.2015.7454321

Benzid, D., \& Kadoch, M. (2018). Raptor code to mitigate Pilot contamination in Massive MiMo. Procedia Computer Science, 130, 310-317. https://doi.org/10.1016/j.procs.2018.04.044

Bogale, T. E., \& Long Bao, L. (2014). Pilot optimization and channel estimation for multiuser massive MIMO systems. Paper presented at the 2014 48th Annual Conference on Information Sciences and Systems (CISS).

Etesami, O., \& Shokrollahi, A. (2006). Raptor codes on binary memoryless symmetric channels. IEEE Transactions on Information Theory, 52(5), 2033-2051. https://doi.org/10.1109/TIT.2006.872855

Farrell, P., \& Honary, B. (2005). Capacity approaching codes design and implementation. IEE Proceedings-Communications, 152(6), 1060-1061.

Jaber, M., Imran, M. A., Tafazolli, R., \& Tukmanov, A. (2016). 5G backhaul challenges and emerging research directions: A survey. IEEE Access, 4, 1743-1766.

Khoueiry, B. W., \& Soleymani, M. R. (2014). Joint channel estimation and raptor decoding over fading channel. Paper presented at the 2014 27th Biennial Symposium on Communications (QBSC). https://doi.org/10.1109/QBSC.2014.6841207

MacKay, D. J. (2005). Fountain codes. IEE Proceedings-Communications, 152(6), 1062-1068.

Majumder, S., \& Verma, S. (2013). Joint channel estimation and decoding of Raptor code on fading channel. Indian Journal of Computer Science and Engineering Vol, 4(2), 168-173.

Marzetta, T. L. (2010). Noncooperative Cellular Wireless with Unlimited Numbers of Base Station Antennas. $\begin{array}{lllll}\text { IEEE Transactions on Wireless } & \text { Communications, } & 9(11), & \text { 3590-3600. }\end{array}$ https://doi.org/10.1109/TWC.2010.092810.091092

Moreira, J. C. e., \& Farrell, P. G. (2006). Essentials of error-control coding. John Wiley \& Sons.

Muaayed, A.-R. (2017). Massive MIMO System: An Overview. International Journal of Open Information Technologies, 5(2), 5-8.

Ngo, H. Q. (2015). Massive MIMO: Fundamentals and system designs. 9175191474. (Vol. 1642): Linköping University Electronic Press.

Shokrollahi, A. (2006). Raptor codes. IEEE Transactions on Information Theory, 52(6), 2551-2567. https://doi.org/10.1109/TIT.2006.874390

Stockhammer, T., Shokrollahi, A., Watson, M., Luby, M., \& Gasiba, T. (2008). Application layer forward error correction for mobile multimedia broadcasting. Handbook of mobile broadcasting: DVB-H, DMB, ISDB-T and media flo, 239-280.

Zirwas, W., Amin, M. B., \& Sternad, M. (2016). Coded CSI Reference Signals for 5G-Exploiting Sparsity of FDD Massive MIMO Radio Channels. Paper presented at the Smart Antennas (WSA 2016); Proceedings of the 20th International ITG Workshop on.

\section{Copyrights}

Copyright for this article is retained by the author(s), with first publication rights granted to the journal.

This is an open-access article distributed under the terms and conditions of the Creative Commons Attribution license (http://creativecommons.org/licenses/by/4.0/). 